\title{
Subminiature eddy current transducers for thickness measurement
}

\author{
Sergey Dmitriev ${ }^{1}$, Vladimir Malikov ${ }^{1, *}$, and Anatoly Sagalakov ${ }^{1}$ \\ ${ }^{1}$ Altai State University, Faculty physics and technology , 65604, Barnaul, Russia
}

\begin{abstract}
A new gage system was made based on the eddy-current transducer. The system makes it possible to evaluate the possibility of using the eddy current method to measure the thickness of conductive and dielectric coatings applied on a conductive base material. One of the important advantages of the developed gage system is the possibility of local measurement of the thicknesses of conductive and non-conductive coatings. In this article, in particular, the measurement features are described in detail, as well as the specificity of the gage system. Also it represents data, showing the dependence of signal amplitude on objects of different thicknesses, and experiments proved the limiting dimensions, at which measuring data are useful.
\end{abstract}

\section{Introduction}

To control the thickness of electrically conductive sheets, films, plates, coatings on them, the walls of pipes, cylindrical and spherical chambers and to determine the air gap between plates of the same material thickness gages based on the eddy current method are used. Eddy-current thickness gauging of the object, which is inherently a dielectric or conductive layer (so-termed, a coating) applied on a conductive base material requires the control, based on the phase measurement of the eddy-current transducer (ECT) signal [1]. This method makes it possible to determine the thickness of the coating $\mathrm{d}$.

For the estimation of coating thickness with known conductivity, Takahashi et al. [2] discussed the feasibility of thickness evaluation of Ni-based alloy coating sprayed on 304 austenitic stainless steels using swept frequency EC (SFEC) testing. The coating thicknesses were estimated within a maximum error of 22 fim through inverse modeling methods. Barbosa [3] presented a formula to obtain the coating thickness of galvanized steel wires from the impedance of a solenoid containing a sample of wires with the assumption that the skin depth of the ECs in the coating is much larger than the coating thickness. Tai et al. [4,5] and Yang [6] used pulsed EC and SFEC techniques to determine the thickness and conductivity of metallic coating on a metal substrate for the case when either the coating or the substrate is magnetic.

Sakran et al. [7] demonstrated a reflection mode EC technique to measure the thickness of conducting layers in a range from 0.1 to $1 \mathrm{im}$ with the spatial resolution of $1-2 \mathrm{~mm}$ at the microwave frequency. Lefebvre and Mandache [8] studied the behavior of the lift-off point

\footnotetext{
${ }^{*}$ Corresponding author: osys11@,gmail.com
} 
of intersection (LOI) under various testing conditions, and LOI is presented to measure the thickness of conductive layers over the ferromagnetic substrates. Zhao et al. [9] experimentally demonstrated the feasibility of the nanometallic film thickness measurement by using the EC method.

Typically, surface ECT is used for these measurements. Such a transducer may have up to three windings: energizing winding (creating an excitation field), measuring winding (designed directly for measurements) and compensation winding designed to reduce the influence of the energizing winding on the final signal. In this case, it becomes possible to use electromotive force in transducer compensation winding as a reference signal, and the phase is measured based on the sinusoidal signal on the energizing winding. This approach is used to improve the measurement accuracy. The electromotive force introduced into the measuring winding has a phase depending on the geometric parameters of the ECT, using the frequency of the current $f$ on the energizing winding. Important parameters influencing the phase are also the gap between the transducer and the coating, the electrical conductivity of the coating $\sigma_{1}$ and the base $\sigma_{2}$, as well as the magnetic permittivity of the base.

Among the problems of this approach is the variation in the electrical conductivity $\sigma_{1}$ at different points of the coating surface of thickness $d$, and when controlling various objects with similar coatings [10]. This causes the oscillations of phase of the electromotive force, which leads to increase in errors when measuring the thickness of the coating. Various types of an offset (amplitude, phase, amplitude-phase) resulting from the influence of electrical conductivity $\sigma_{1}$ in this case practically have no effect. Underfrequency $f$ of excitation of current on the energizing winding makes it possible to reduce the effect of electrical conductivity. However, in this case, the substrate thickness and the magnetic permittivity of the substrate become an important influence factor.

Taking in consideration the abovementioned factors, it is necessary to choose such a frequency of an instrument to offset from oscillations of electrical conductivity, while not permitting the influence of the magnetic permittivity of the substrate. Optimal in this case is to measure not the phase $\varphi$, but the amplitude A of the signal, on which the substrate parameters have lower effect, rather than on the phase.

Therein when measuring the amplitude of ECT signal for the purpose of measuring the coating thickness, a certain value of electrical conductivity is recognized.

The purpose of this work was to estimate the application possibility of the amplitude eddy-current method alone in order to determine the thickness of the conductive or dielectric coating, placed on the conductive base, as well as to estimate such measurements inaccuracy. The conducted research showed the possibility of the amplitude eddy-current method application to detect the local thick-ness of the conductive objects, represented by several alternating conductive and non-conductive layers and solid conductive objects.

\section{Description of the system}

A subminiaturized eddy-current transducer $[11,12]$ is designed for the local examination of the different coatings thickness in experiments, as well as to reveal the influence of different coatings on the output signal quantity. In contrast to existing sensors, it permits local measurements with sections measuring a few microns, to depths of the order of $5 \mathrm{~mm}$. The electrical conductivity of the alloy is directly measurable, and its distribution over the sample surface and thickness may be readily established. The eddy-current method is based on the dependence of the current magnitude and distribution on the geometric and electromagnetic parameters of the sample and on the mutual position of the sensor and the sample. The basic informative parameter $\beta_{0}$ is a generalized characteristic of the object, the eddy-current sensor, and the frequency of the electromagnetic field. We need to develop an 
adequate model of the response of a eddy-current transformers of plate type, which are sensitive to many variables and permit reproduction of the voltage hodograph at small values of $\beta_{0}$. We have plotted hodographs illustrating the influence of various parameters on the induced voltage on the basis of the proposed model [13-15]. The eddy-current transformer is connected to the sound board of a computer with special software that controls the voltage at the transformers exciting winding and also reads the voltage at the measuring winding (in arbitrary units). On the basis of preliminary calibration, these readings are converted to values of the electrical conductivity. The exciting winding (diameter $\mathrm{D}_{1}=0.12-0.13 \mathrm{~mm}$ ) of the superminiature eddy-current transformer consists of ten turns of copper wire (cross-sectional area $5 \mu \mathrm{m}^{2}$ ). The measuring winding (diameter $0.05-0.08 \mathrm{~mm}$ ) consists of 130 turns of copper wire (cross-sectional area $20 \mu \mathrm{m}^{2}$ ). To minimize the influence of the exciting winding on the final signal, the circuit includes a compensation winding that consists of 20 turns of copper wire (crosssectional area $5 \mu \mathrm{m}^{2}$ ), connected to the measuring winding in such a way that the voltage of the exciting winding is subtracted from the result. The windings are wrapped around a core of $2000 \mathrm{NMZ}$ ferrite (relative magnetic permeability $\mu_{\max }=500$ or else of $81 \mathrm{NMA}$ alloy annealed by a special method (if greater localization of the magnetic field is required). The core consists of a tetrahedral pyramid (height $1 \mathrm{~mm}$ ), with a square base (sides of $0.2 \mathrm{~mm}$ ). The measuring winding rests on the points of the pyramid, which improves the localization of the magnetic field.

The eddy-current transducer (Figure 1) is a transformer with measuring (1), exciting (2), and compensation (3) windings and a magnetic circuit 4, which is located inside the cylindrical platform 5 with tracks that are cut on the external side for windings. The platform is impregnated with a compound 6 at a temperature of $200^{\circ} \mathrm{C}$ to prevent the disintegration of the windings when the ferrite screen 7 , which is intended for the localization of the electromagnetic field on the tested object, is put in place. From the outside the transducer is contained in a corundum washer 8 , which protects the core 4 from contacting the tested object.

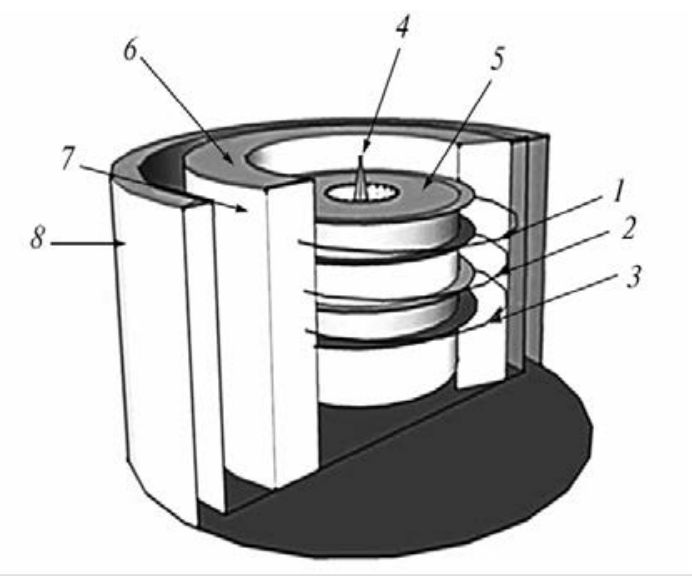

Fig. 1. Scheme of eddy current transducer.

Such transformers permit effective localization of the magnetic field, so that defects as small as $250 \mu \mathrm{m}$ may be detected. In addition, the magnetic field penetrates into the sample to a considerable depth when working at relatively low frequencies. The corresponding software is written in the language $\mathrm{C}++$ for the Windows operating system. By means of the Windows mixer subsystem, the software controls the voltage applied to the exciting winding, thereby specifying the amplitude and frequency of the sinusoidal digital signal from the virtual generator. 


\section{Operation of the measuring system}

The measuring system, which is based on a miniature eddy-current transducer, operates as follows. The software of the personal computer controls the operation of the generator, which produces a train of rectangular voltage pulses with the repetition rate $f$ that is necessary for the operation of the eddy-current transducers. This voltage is sent through a preamp to the microphone input of the sound card and then to the input of the analogdigital converter in the sound card. The resulting digital signal is sent to the analysis and control module of the software. This module determines the magnitude of the digital signal (in conventional units) corresponding to the voltage $U_{m}$ at the measuring winding.

The computer's sound card permits variation in frequency of the electromagnetic field created by the exciting winding within the range $100-4000 \mathrm{~Hz}$ in the course of scanning.

\section{Results and discussion}

To test the new gage system the scanning of an aluminium coating applied on a copper base material was performed. Measurements were made at a frequency of $700 \mathrm{~Hz}$. The coating had different thickness, and the thickness of the copper base was 3 millimetres.

Figure 2 shows the dependence of the signal value on the thickness of the aluminium coating on a non-ferromagnetic base material. In case of an increase in the thickness of the coating to the value of $1200 \mu \mathrm{m}$, the signal decreases from 28 to $22 \mathrm{mV}$, therein at values from 750 up to $1500 \mu \mathrm{m}$, the signal is smaller than the monolith signal, this, in turn, demonstrates that the thickness of the coating is not large enough. Whereas, with a dielectric thickness of 1500 and up to $2500 \mu \mathrm{m}$, the signal value is constant and completely corresponds to the readings of the field value from the monolith.

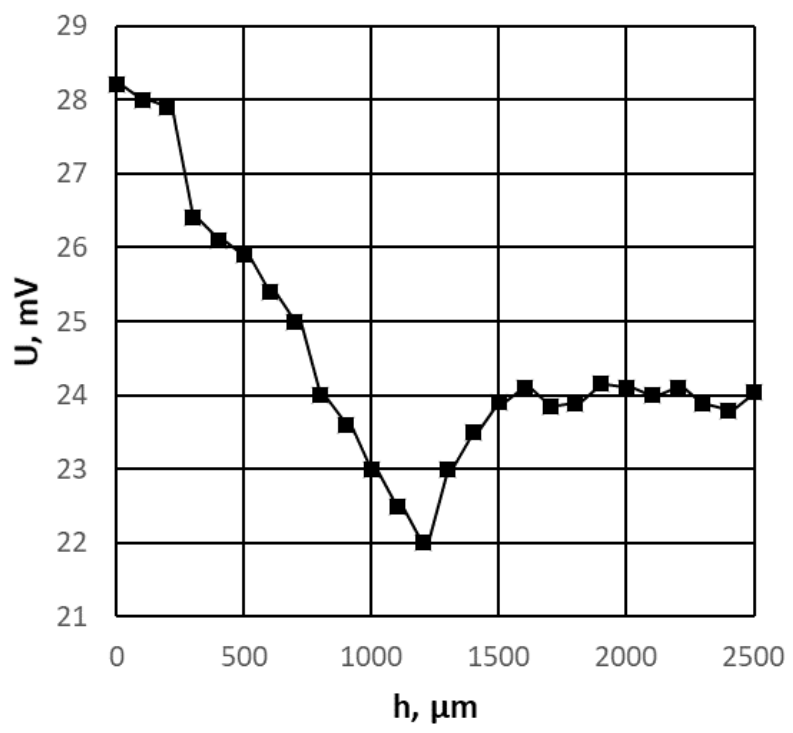

Fig. 2. Dependence of the signal value on the thickness of an aluminium coating applied on a copper base.

The next experiment demonstrates the dependencies illustrating the signal change from the thickness of the laminated coating with alternating foil and polyethylene layers, placed 
on the copper base. The object under study was represented by alternating aluminium foil $(20 \mu \mathrm{m})$ and polyethylene $(20 \mu \mathrm{m})$ layers.

Figure 2 demonstrates the dependency of the signal intensity on the thickness of the laminated coating with alternating foil and polyethylene layers, placed on the copper base. The thickness being within the range of 0 to $100 \mu \mathrm{m}$, the signal quantity from the base changes from 29 to $26 \mathrm{mV}$, while the thickness being 100 to $240 \mu \mathrm{m}$, the pattern of the signal change is flatter. In the range of 240 to $400 \mu \mathrm{m}$, the signal changes from 26 to 23 $\mathrm{mV}$, which is stipulated by the contribution of the signal from the laminated coating and the decrease in the contribution of the signal from the copper base.

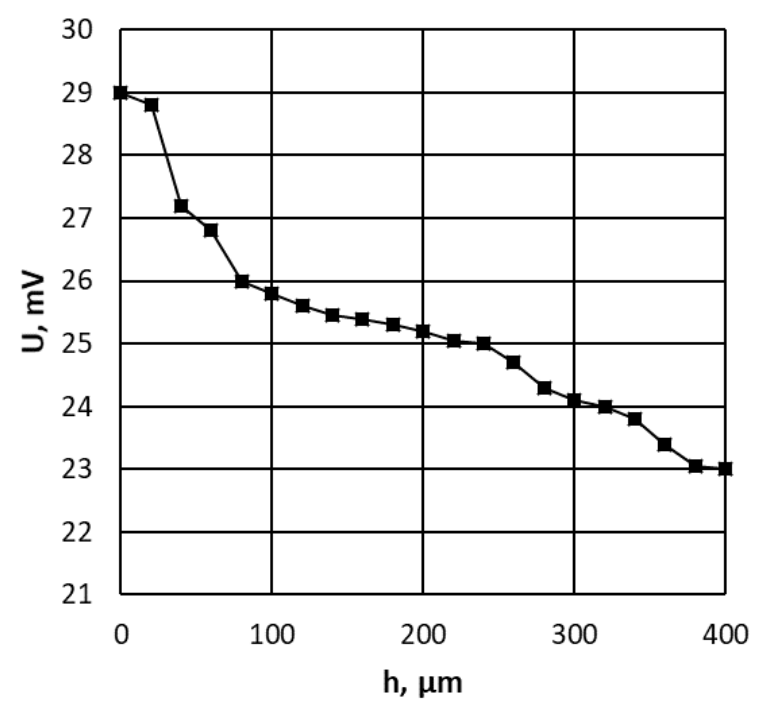

Fig. 3. Dependence of the signal intensity when scanning the laminated structure

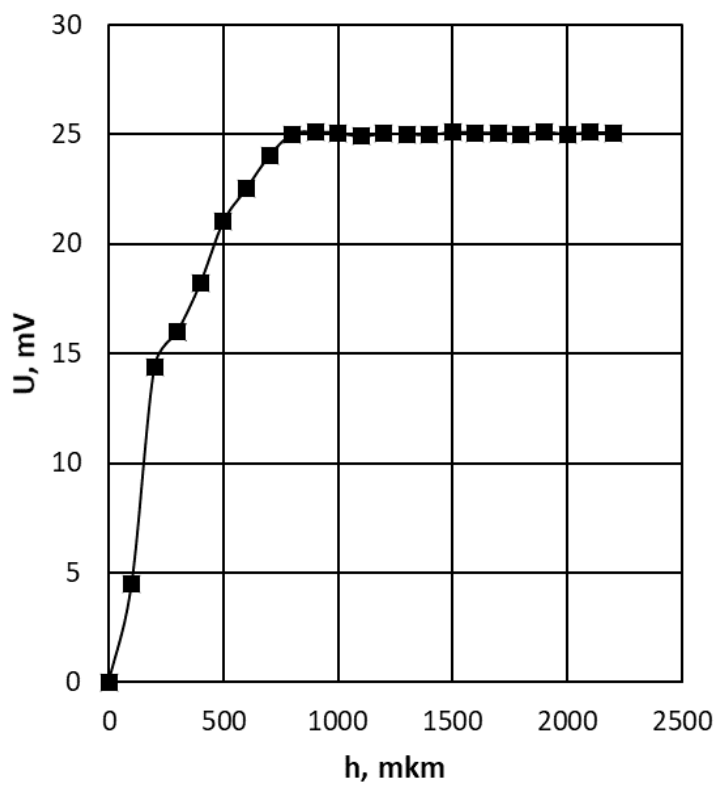

Fig. 4. Dependence of the signal on the thickness of the sample of aluminium 
In the third test experiment, we conducted the scanning of a sample being by nature of a solid object made of aluminium having different thickness. Figure 3 shows the dependency of the signal quantity on the thickness of the aluminium sample. The contribution of the sample deeper layers to the signal amplitude rises with the increase in the sample thickness. The thickness being changed from 100 to $1200 \mu \mathrm{m}$, the signal quantity increases from 7 to $25 \mathrm{mV}$. If the thickness is changed from 1200 to $2200 \mu \mathrm{m}$, the signal quantity is stable and corresponds to the amplitude value from the solid unit $(25.5 \mathrm{mV})$. In the final study, the experiment was also carried out with copper samples and paint coating. A layer of paint was applied to the pre-cut samples of copper. Measurements were made at a frequency of $400 \mathrm{~Hz}$.

As it can be seen from the dependence of the signal amplitude on the thickness of the dielectric coating (Fig. 4), an output signal quantity decreases rapidly with increasing of coating thickness. This dependence can be approximated by an exponential function:

This dependence of the approximating function are graphically shown in Fig. 4. As can be seen from the figure, the received signal decreases exponentially with increasing of thickness of the dielectric coating.

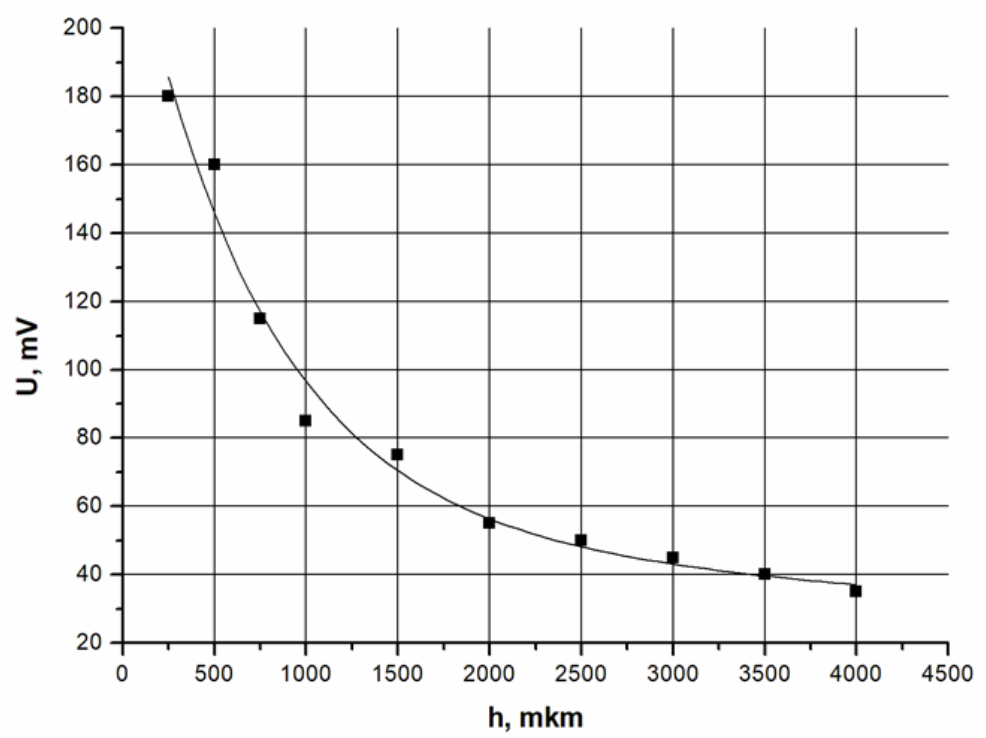

Fig. 5. The response value during the scanning in the area of two slabs junction. A1 - A2 correspond to the edges of the area where the junction presence influences the inserted voltage.

\section{Conclusions}

The presented gage system was used for the study of objects representing conductive and non-conductive coatings placed on a conductive basis, as well as for measuring the thickness of monolithic conductive objects. We assessed the possibility of local determination of the thickness of conductive and dielectric coatings using the amplitude of the signals of ECT.

It is found that the thickness of the coating affects the signal of the eddy-current transducer. This hereafter allows the use of the amplitude method to control objects of a similar class for local measurements of the thickness of conductive and non-conductive coatings, as well as other objects. 


\section{References}

1. P. Noimaier, In the world of nondestr. test., 2(40), 29 (2008)

2. Y. Takahashi, Int. J. Appl Electromagn. Mech., 39(1), 419 (2012)

3. C. F. Barbosa, IEEE Trans. Magn., 50(7), 1 (2014)

4. C. C. Tai, J. H. Rose, M. Lechuga, J. C. Moulder, Rev. Sci. Instrum., 67(11), 3965 (1996)

5. C. C. Tai, Rev. Sci. Instrum., 71(8), 3161 (2000)

6. H. C. Yang, C. C. Tai Meas. Sci. Technol., 13(8), 1259 (2002)

7. F. Sakran, M. Golosovsky, D. Davidov, Appl. Phys. Lett., 78(11), 1634 (2001)

8. J. Lefebvre, C. Mandache Int. J. Appl. Electromagn. Mech., 27(1-2), 1 (2008)

9. Q. Zhao, Q. Yu, Z. Qu, L. Si, X. Lu, Proc. IEEE Instrum. Meas. Technol. Conf., 1 (2011)

10. A.S. Bakunov, V.A. Kaloshin, Contr. diagn., 211, 27 (2016)

11. S. F. Dmitriev, A. O. Katasonov, V. N. Malikov, A. M. Sagalakov, IOP Conf. Ser.: Mater. Sci. Eng., 71, 1 (2015)

12. S. Dmitriev, L. Dmitrieva, V. Malikov, A. Sagalakov, IOP Conf. S.: Mat. Sci. Eng. 116, 1 (2015)

13. S. F. Dmitriev, V. N. Malikov, A. M. Sagalakov, A. V. Ishkov, A. O. Katasonov, Adv. Intel. Sys. Comp., 692, 655 (2017)

14. S. F. Dmitriev, A. V. Ishkov, V. N. Malikov, A. M. Sagalakov, Measur. Techn., 60(4), 372 (2017)

15. S. F. Dmitriev, A. V. Ishkov, V. N. Malikov, A. M. Sagalakov, Mat. Sci. Forum, 906, 147 (2017) 